Bangladesh Journal of Neuroscience 2015; Vol. 31 (2): 102-109

\title{
Effects of Selective Rehabilitation on Neck Pain due to Cervical Spondylosis - A Clinical Trial
}

\author{
MD. ABDUS SHAKOOR ${ }^{1}$, SHAMSUNNAHAR ${ }^{2}$, NAYEEM ANWAR ${ }^{3}$, MD. MUHIBBUR RAHMAN $^{4}$, \\ FATEMA ZOHRA ${ }^{5}$, MD. MOYEENUZZAMAN ${ }^{1}$
}

\begin{abstract}
:
Background:Pain in the neck is a common complaint of the patients attending the hospital. In clinical practice, neck pain is seen frequently as a presenting symptom and sometimes it becomes disabling and compromises the working capacity. One of the most common causes of pain in the neck is cervical spondylosis. Rehabilitation treatment may play an important role to improve the condition of the patients. For this purpose, the study was done to find out the effects of rehabilitation treatment on chronic neck pain to improve the present situation regarding treatment. Methodology: A randomized clinical trial was conducted in the department of Physical Medicine and Rehabilitation, Bangabandhu Sheikh Mujib Medical University, Dhaka, Bangladesh. A total of 150 patients were included and they were divided into two groups: group-A and group-B. Group-A was treated with selective rehabilitation and Group $-B$ was treated with NSAID only. History, clinical examination and relevant investigations were done. The findings were recorded at first attendance and follow up was done weekly for six weeks. The results were expressed as mean $\pm S D$ and the level of significance was expressed by p-value unless otherwise stated. Student's 't' tests was done to test the hypothesis. Results: Among the study subjects 48(32\%)were male and 102 (68\%) were female. The male female ratio was 1: 2.12. There was significant improvement in both the group after treatment $(P=0.001)$. But in comparison between two groups, all the baseline criteria were identical. There was no significant improvement between two groups up to $5^{\text {th }}$ weak $(P>05)$ but significant improvement was seen in Group- $B$ than Group-A after six weeks treatment $(P=0.03)$. This results indicates that the improvement of the patient with cervical spondylosis was seen in selective rehabilitation group and in NSAIDs group. And improvementwas same in both the group up to $5^{\text {th }}$ week and after six weeks more improvement was found in NSAIDs group. Conclusions: By this study, it may be concluded that to reduce symptom and disability, rehabilitation treatment can be used effectively for the treatment of chronic neck pain without analgesics and by this way nephropathy due to NSAIDs can be avoided.
\end{abstract}

Key words:Selective rehabilitation, neck pain, cervical spondylosis.

Introduction:

Pain in the neck is a common complaint of the patients attending the hospital. In clinical practice, neck pain is seen frequently as a presenting symptom and sometimes it becomes disabling and compromises the working capacity. Many of the patients come to the department of Physical Medicine for proper treatment and rehabilitation. Most of them are suffering from cervical spondylosis.Osteoarthritis is the most common

1. Professor, Physical Medicine and Rehabilitation, Bangabandhu Sheikh Mujib Medical University, Shahbagh, Dhaka-1000, Bangladesh.

2. Professor and Chairman, Physical Medicine and Rehabilitation, Bangabandhu Sheikh Mujib Medical University, Shahbagh, Dhaka-1000, Bangladesh.

3. HMO, Department of Neurology, Bangabandhu Sheikh Mujib Medical University, Shahbagh, Dhaka-1000, Bangladesh.

4. Resident, Physical Medicine and Rehabilitation, Bangabandhu Sheikh Mujib Medical University, Shahbagh, Dhaka-1000, Bangladesh.

5. HMO, Physical Medicine and Rehabilitation, Bangabandhu Sheikh Mujib Medical University, Shahbagh, Dhaka-1000, Bangladesh. 
rheumatological disease that affects more than $80 \%$ of the population aged 55 years and older ${ }^{1}$. Cervical spondylosis is one kind of osteoarthritis, disc degeneration of the intervertebral disc with associated osteophyte formation ${ }^{2}$. Degenerative changes in the cervical spine (cervical spondylosis) may be associated with neck pain but usually only when the degenerative changes are severe. Mild or moderate degenerative changes are often seen in asymptomatic individuals. In general, the pain of mechanical disorders is intermittent and related to use. Neurological examination will often reveal the level of entrapment ${ }^{3}$. Neck stiffness existed as a common disorder in the age group of 25 to 29 years in our working population and 25 to 30 percent had one or more attack of stiff neck. For working population over 45 years of age, this figure rises to 50 percent. Brachial neuralgia occurs later in life than stiff neck does with a frequency of 5 to 10 percent in the 25 to 29 years' age group, rising to 25 to 40 percent after the age of 45 years and overall $45 \%$ of working men experience at least one attack of stiff neck, $23 \%$ report at least one attack of brachial neuralgia and $51 \%$ suffer from both symptoms ${ }^{4}$. Pain and stiffness in the neck can originate from many tissue sites and can result from a number of mechanisms. One of the most common causes of pain and disability in the neck and arm is cervical degenerative arthritis (cervical spondylosis) ${ }^{5}$. Cervical spondylosis is a clinical syndrome in which cervical spine degenerates to such an extent that symptom arises ${ }^{6}$. It is characterized by osteophytosis, narrowing of intervertebral joint spaces and foramina and compression of the nerve roots and spinal cord.

It runs a prolonged course with intermittent periods of relief. It commonly affects people above the age of 40 and is responsible for varying grades of disability ${ }^{7}$. It may produce clinical symptoms and I or signs and these are very variable, ranging from neckache, with or without headache, to brachialgia, myelopathy and vertebro-basillar insufficiency and indeed there may be various combinations and permutations of one or more of these in the individual patients. A study was carried out by Alam et al. in 1995 in the Rheumatology clinic in the department of Physical Medicine, IPGMR and they showed that cervical spondylosis was the commonest lesion $(23.5 \%)$ amongst the various rheumatic disorders ${ }^{8}$. However, there are few studies were found to see the effects of rehabilitation program. The use of physical therapies e.g. Transcutaneous Electrical Nerve Stimulation, other electromagnetic applications, exercises and aids \& appliances are widely used by pain specialist 9,10 . Several types of physical therapy has potential effects on musculoskeletal pain $11,12,13$. Cervical traction is widely used in treating various types of neck disorders, opinions are divergent on methods of application and clinical results ${ }^{14}$. But rehabilitation treatment may play an important role to improve the condition of the patients. This large number of patients should be managed properly for improvement of their working capacity and thus they may be able to contribute themselves for the prosperity of the country. For this purpose, the study was done to find out the effects of rehabilitation treatment on neck pain due to cervical spondylosis to reduce disability \& recurrence of symptoms.

\section{Objectives:}

The objectives of this research were to observe the effects of selective rehabilitation on cervical spondylosis

and comparing the effects of rehabilitation with that of drug therapy.

\section{Materials and Method:}

The patients having chronic cervical spondylosis were selected from the department of Physical Medicine, Bangabandhu Sheikh Mujib Medical University( BSMMU). A total 170 patients aged from 30 to 60 years were selected for the study as per the selection criteria. Selection was done randomly by the way of lottery. On arrival at the department, detailed history was taken and clinical examination was carried out properly. The patient was selected based on the criteria used in the trial of 1966 with some modification, sponsored by the British Association of Physical Medicine. Chronic pain in the neck and arm, the symptoms having a root distribution and being associated with limited and painful movement of the neck, chronic pain in the neck and arm of full root distribution with paresthesia 
but without clinical evidence of abnormality in the neck and chronic pain and stiffness of the neck with or without any rootdistribution (mild) were included.Patients with acute / severe pain and stiffness of the neck or local lesion like rotator cuff tears, tennis elbow and carpal tunnel syndrome, abnormal neurological signs indicating cord compression, definite disorders of the cervical spine, such as rheumatoid arthritis, tuberculosis or any infection, or has a bony injury to the cervical spine were excluded. Before admission into the trial, informed consent of the patients was taken. History, clinical examination and relevant investigations were done. The findings were recorded at first attendance and followed up was done weekly for six weeks. They were divided into two groups by the way of lottery. In group-A, 72 patients were treated with selective rehabilitation program that is - exercise (isometric neck muscle exercisefor 10 repetitions twice daily), cervical collar, neck support, manual cervical traction at home, warm moist compression and instruction in posture while in Group-B, 78 patients were treated with NSAIDs with omeprazole coverage only. Cervical traction was given by a manual home cervical traction set in sitting position. The angle of pull was $15^{>}$flexion of the cervical spine. The traction was given continuously for 20 minutes every day. The weight of traction in the trial was 15 $\%$ (Approx.) of the subject's body weight. Posture correction regarding sitting, lying, reading, writing, shaving, chooking bending etc. was clearly explained to the patients. A cervical collar was prescribed for all the subjects for posture correction and it was used during activity but not during the time of sleeping. All the patients participated in the clinical trial were provided with vitamine- $B_{1}$ (Thiamin) three times daily and NSAID were provided in the Group-B. NSAIDs were given in the form of Naproxen (250 mg) twice daily after meals and Omeprazole (20 mg) was given twice daily to prevent drug induced peptic ulcer. The following tools were used for comparing the treatment:Physician's assessment of the severity of the conditions-pain score, tenderness index \&pain frequency score and patient's assessment of pains by visual analogue scale. The numerical data was analyzed statistically. The results were expressed as mean \pm SD and the level of significance was expressed by $p$-value unless otherwise stated. Statistical analysis was done by using SPSS package for Windows. Student's ' $t$ ' tests will be done to test the hypothesis.

\section{Results:}

A total of 175 patients were selected but 150 patients with cervical spondylosis duly participated in the study. Twenty-five patients were dropped out from the study because they did not take treatments allocated to them and/or could not attend regularly and did not follow advice of the investigators. Among the subjects there were 48(32\%) male and 102 (68 $\%$ ) female (Fig. No-1).

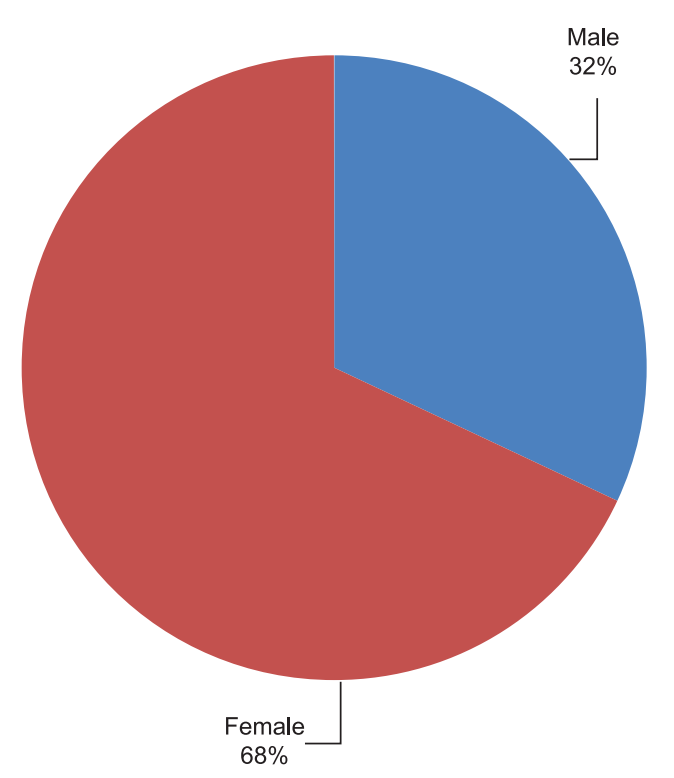

Fig. -1: Sex distribution of the patients $(N=150)$

The male female ratio was 1: 2.12 . Most of the subjects were married (96.7\%). Maximum patients were in the middle class (64\%). The mean age of the subjects was $42.67 \pm 8.85$ years. The highest number of patients was in the $40-49$ years' age group (Fig No-II). And maximum female persons were affected in their earlier age than male (Fig NoIII). Regarding occupation of the patients, maximum patients of cervical spondylosis were house wife 79 $(52.70 \%)$ and the private was in the second position (14\%). 


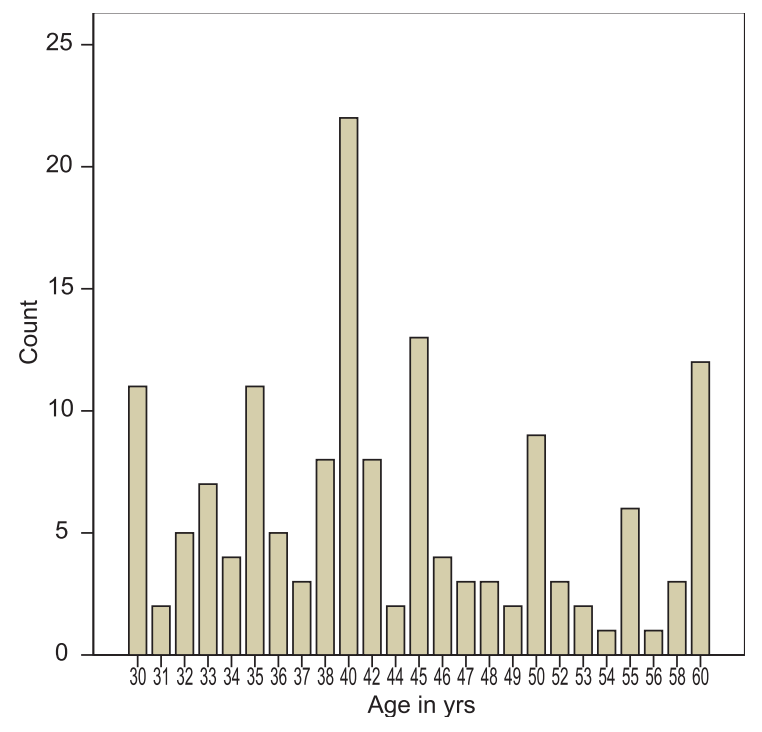

Fig.-2: Age distribution of the patients $(N=150)$.

\section{Results of the clinical trial:}

Base lines characteristics of the patients in both the Group were identical. This result showed that there was no significant deference between two groups regarding baseline clinical characteristics (Table No-I).

In group $-\mathrm{A}$, there were 85 patients initially, but 13patients were dropped out from the study because of their irregularities to take treatments. There were 72 patients participated in the clinical trial and they took treatments and all suggestions properly. There

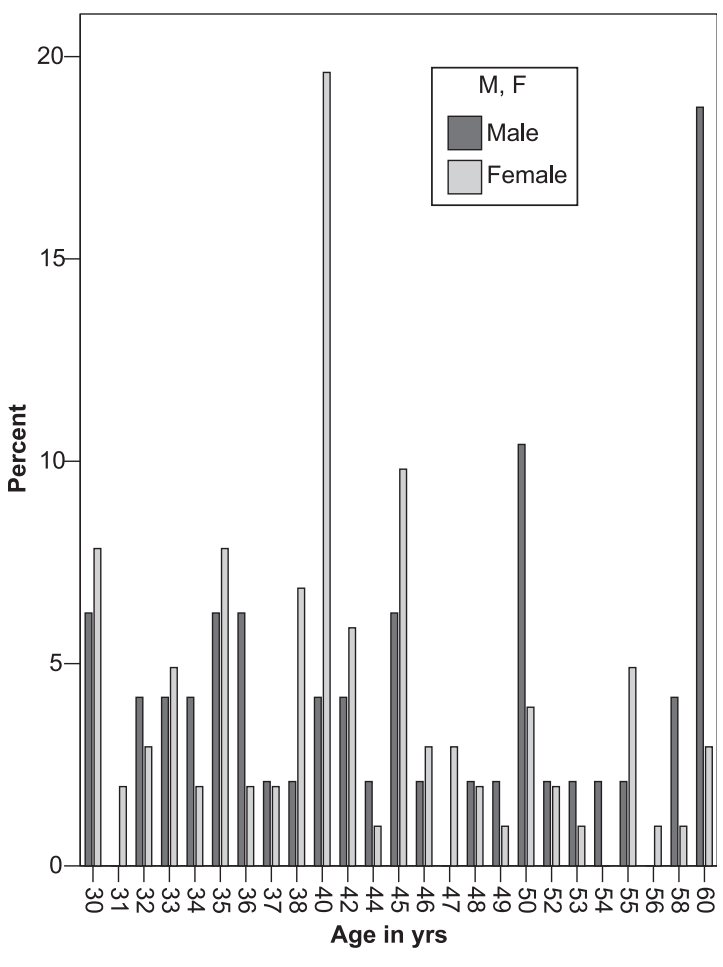

Fig.-3: Age and sex distribution of the patients $(N=150)$.

was marked improvement of the condition of the patients in response to treatment for 6 weeks. The numerical data of pretreatment and after treatment assessment scores were compared statistically (paired student's ' $t$ ' test) and found highly significant $(P=0.001)$. So selective rehabilitation was found significantly effective to reduce the sign \& symptoms of cervical spondylosis. In group - B, there were 88 patients initially, but 10 patients were dropped out from the study because of their irregularities to take treatments. There were 78 patients participated in

Table-I

Distribution of baseline clinical characteristics of the patients included in the clinical trial.

\begin{tabular}{lcccccc}
\hline Groups & Age in years & Pulse / $\mathrm{m}$ & $\begin{array}{c}\text { Systolic BP } \\
(\mathrm{m} \mathrm{m} \mathrm{of} \mathrm{Hg})\end{array}$ & $\begin{array}{c}\text { Diastolic BP } \\
(\mathrm{m} \mathrm{m} \text { of Hg })\end{array}$ & Height in Cm & Weigut in Kg \\
\hline Group -A $(\mathrm{n}=72)$ & $43.08 \pm 8.39$ & $76.90 \pm 4.27$ & $127.71 \pm 10.47$ & $81.25 \pm 6.32$ & $157.93 \pm 7.01$ & $63.04 \pm 6.49$ \\
Group -B $(\mathrm{n}=78)$ & $42.29 \pm 9.30$ & $76.49 \pm 4.84$ & $126.03 \pm 12.41$ & $79.81 \pm 7.70$ & $157.46 \pm 7.57$ & $61.84 \pm 6.46$ \\
$p-$ value & 0.58 & 0.47 & 0.38 & 0.24 & 0.69 & 0.26 \\
$95 \% \mathrm{Cl}$ & -2.06 to 3.64 & -1.05 to 1.88 & -2.01 to 5.38 & -0.82 to 3.71 & -1.88 to 2.82 & -0.89 to 3.28 \\
\hline
\end{tabular}

$\mathrm{n}=$ Number of patients,

Results are expressed in mean \pm standard deviation

$p=0.05$ is considered the level of significance.

$\mathrm{Cl}=$ Confidence interval 
the clinical trial. There was improvement of the condition of the patients in response to treatment for 6 weeks. The numerical data of pre-treatment and after- treatment assessment scores were compared statistically (paired student's ' $\mathrm{t}$ ' test) and found highly significant $(p=0.001)$. So NSAIDs were found also effective to reduce the sign \& symptoms of cervical spondylosis. At the time of first visit, there was no significant improvement between two groups up to $5^{\text {th }}$ weak $(P>05)$ but on the other hand there was significant improvement in Group-B than Group-A after six weeks treatment $(P=0.03$, Table No-2). This result indicates that the improvement of the patient with chronic cervical spondylosis was seen in selective rehabilitation group and also in NSAIDs group. And improvement is more or less same in both the group upto $5^{\text {th }}$ week and after six weeks more improvement was found in NSAIDs group $(P=0.03)$.

Table-II

Distribution of comparative improvementbetween two groups.

\begin{tabular}{lcc}
\hline & $\begin{array}{c}\text { pre-treatment } \\
\text { scores }\end{array}$ & $\begin{array}{c}\text { post-treatment } \\
\text { scores }\end{array}$ \\
\hline Group-A $(\mathrm{n}=72)$ & $12.89 \pm 3.38$ & $6.69 \pm 3.12$ \\
Group-B $(\mathrm{n}=78)$ & $13.76 \pm 3.83$ & $5.65 \pm 2.70$ \\
$p$-value & 0.14 & 0.03 \\
$95 \% \mathrm{Cl}$ & -2.03 to 0.29 & 0.09 to 1.98 \\
\hline
\end{tabular}

$\mathrm{n}=$ Number of patients, results are expressed in mean \pm standard deviation;

$p=0.05$ is considered the level of significance. $\mathrm{Cl}=$ Confidence interval

\section{Discussion:}

Cervical spondylosis may affect people of wide range of groups. In our study maximum number of patients was in the $40-49$ years age group (35.67\%). The mean age of the patients in our study was found $42.67 \pm 8.85$ years. Bhattecharjee $B \mathrm{~N}$ et al. found, in their study on cervical spondylosis that maximum number of patients in the 40-49 years, which favoursthe same result found in our study. However, their sample size was small and they selected patients from different hospitals of Dhaka city. On the other hand, study of British Association of Physical Medicine showed most patients fell in the 40 to 60 years age group, which is also favourable to our study ${ }^{15}$.Among the subjects there were
48(32\%) male and $102(68 \%)$ female. The male female ratio was 1:2.12. But The British Association of Physical Medicinefound 203 male and 290 female patients during their study, where the male female ratio was $1: 1.4^{15}$. This is nearer to our study. The more female in our study may be due to awareness and increasing female education. Irvine et al. studied almost equal number (223 females and 230 males) of matched male and female patients and found slight preponderance of male over females ${ }^{16}$. The figure of this series does not reflect male-female ratio of general population because female patients attend hospitals less often because of shyness and social custom. If the general population could be studied, the number of female patients would be gone up.Regarding occupation of the patients, maximum patients of cervical spondylosis were house wife $79(52.70 \%)$ and the private was in the second position $14 \%$. Highest number of patients seen in our study was housewife but Bhattecharjee $\mathrm{B} \mathrm{N}$ et al. found that highest number of patients was table worker and housewife was second 17 . This may be due to more female participants in our study. The number of patients in this series can be attributed to the fact that prolonged neck flexionextension movement during their household works. Most of our middle aged and elderly female patients have no occupation other than housewife. This may be important for the highest incidence of neck pain among housewives.

Treatment response: In our study, significant improvement was observed in response to selective rehabilitation, there was significant difference between pre-treatment and after treatment. The result is in the line with the results presented by the British Association of Physical Medicine ${ }^{15}$. They found that during traction relief of their symptoms was obtained in 105 of the 114 patients (92\%). Bhattacharjee B.N et al. found in their study that cervical traction has no significant improvement but their sample size was only 18 in cervical traction group but our sample size was 100 in number and he used only cervical traction without exercise but we use isometric neck muscle exercise along with cervical traction. But Swezey R.L et al found significant improvement in response to home cervical traction ${ }^{18}$. They used simple, in-expensive over- 
the-door home cervical traction method of treatment requiring 5 minutes of cervical traction twice daily for both cervical pain and radiculopathy syndromes. They found over-the-door home cervical traction modality provided symptomatic relief of $81 \%$ of the patients with mild to moderately severe cervical spondylosis syndromes, which supports the findings of our study because we used home cervical traction daily for 15 minutes. On the other hand Wareham T et al found that very good results in their study, where 100 patients treated with cervical traction with radiculopathy, 90 obtained complete relief within about half a minute of the application of the traction 19. Cervical traction is also effective to reduce deformity of the cervical spine. Graziano G.P et al. found that excellent correction of deformity and radiographic union were achieved in all patients with severe cervical deformity in rheumatoid arthritis ${ }^{19}$. Honet C.J et al found good to excellent results in $92 \%$ of patients treated with cervical traction ${ }^{21}$. This supports the results of our study also. Caldwell et al. treated 577 patients of cervical syndrome and found $82 \%$ good results with constant traction on an average of 13 session treatments, $92 \%$ exhibit good and $8 \%$ moderate improvement and there was no failure ${ }^{22}$. Shakoor et al. also found better improvement of cervical spondylosis with cervical traction in the hospital setting 23,24 . This is also in favour of our study we also used constant traction. The only effects which traction can be expected to achieve are some distraction between vertebrae at the intervertebral disc and apophyseal joints, tensing the longitudinal ligaments of the spinal column, and some slight widening of the intervertebral foramina ${ }^{23}$. The two general objectives in

applying cervical traction are (1) to stretch the posterior cervical region and (2) to enlarge the interspaces at the intervertebral foramina ${ }^{14}$. Probably due to this distraction there was good results we obtained in our study. In a study in the department of Physical Medicine \& Rehabilitation, IPGM\&R, Dhaka, it was found that there was significant improvement in response to cervical traction group, where cervical traction was given along with exercise and NSAIDs ${ }^{23}$. But in our study we used traction without NSAIDs.
In the study of Caldwell J.W et al. neck muscle exercises were applied and found good results ${ }^{21}$. In our study, we used isometric neck muscle strengthening exercises and we found significant improvement. This indicates that exercise also played an important role to improve the condition. In some studies, it was found that strength training was effective moderately to reduce pain and improving function and stiffness in osteoarthritis 25 More specific exercises were used to strengthen muscle to increase range of motion of a joint to reeducate patients in appropriate use of joints and reduce pain. Exercises to stretch the involved muscles are the key to sustained relief of myofascial pain ${ }^{26}$. Restoration of normal muscular length, posture and muscle overwork. improved conditioning (exercise tolerance or stamina) and increased strength through exercises, reduces the likelihood of their developing trigger points. A carefully graded exercise programme is needed to increase endurance. Strengthening exercises are recommended, which should be done smoothly and slowly, with a certain number of repetitions to achieve conditioning ${ }^{27}$. In our study we used certain number of repetitions (10 times) twice daily and found statistically significant improvement. However, we used cervical traction along with exercise, ADL advice and cervical collar as selective rehabilitation. Education interventions can be split into two main areas: providing information on the disease and how to alleviate the symptoms through behavioral changes (physical coping) or reducing symptoms through physiological strategies (physiological coping) ${ }^{25}$. In a study, Tanaka $S$ et al. found significant relationship of activities of daily living on muscle atrophy in patients with joint disorders 27 . Chard $\mathrm{J}$ et al. found that there are moderate improvement of pain and function due to posture correction without side effects ${ }^{25}$. We applied

posture correction procedures in both the groups and found insignificant improvement regarding pain and range of motion. We also used cervical collar (one kind of brace) for maintaining good neck posture during working period, which also supported by Chard $\mathrm{J}$ et al. The results are in the line of Tanaka $\mathrm{S}$ et al. and Chard $\mathrm{J}$ et al.Education of the patient about the pain and the perpetuating and aggravating 
factors is of major importance. It helps in the programme and allows satisfactory long-term results.

We found significant improvement in response to treatment with NSAIDs. We used tablet naproxen (250 mg) twice daily, as all the subjects were chronic in nature. Chard $\mathrm{J}$ et al. found in a review that NSAIDs were highly effective for pain and function but with potential side effects ${ }^{25}$. This is in favour of our study. NSAIDs therapy is the most prevalent category of adverse drug reaction. Thus NSAIDs may be used to reduce pain and improve function but it should be used very cautiously.

Comparative study: We compare between selective rehabilitation and non-steroidal antiinflammatory drugs and found significant improvement in NSAIDs group than rehabilitation group after six weeks $(p=0.03)$ but there was no significant improvement throughout the study upto $5^{\text {th }}$ week. But in the present study no NSAIDs was given to the selective rehabilitation group. In spite of that there was significant improvement after treatment in that group. In this study, NSAIDs were given in both the groups but cervical traction plus NSAIDs showed good results. In both the studies it was found that cervical traction is superior to other form of treatment, which also supports the results obtained in the present study because without giving NSAIDs same improvement was found in rehabilitation group upto $5^{\text {th }}$ week.. On the other hand there were potential side effects of NSAIDs rather than physical therapies ${ }^{25}$. Gastrointestinal (G I) complications related to NSAIDs therapy are the most prevalent category of adverse reaction ${ }^{28,29}$. These side effects includes -dyspepsia, gastric ulcer, perforation, gastric outlet obstruction, major or minor acute or chronic $\mathrm{Gl}$ bleeding even death 29,30 . But rehabilitation has negligible side effects. In a study, Dabbs $V$ et al. found that cervical manipulation for neck pain is much safer than the use of NSAIDs, by as much as a factor of several hundred times and there is no evidence that indicates

NSAIDs use is any more effective than cervical manipulation for neck pain. So NSAIDs should be used very cautiously. And if it is possible to avoid NSAIDs we should avoid it and go for nonpharmacological therapy like rehabilitation treatment.
Our study has it's obvious limitations. It was confined to a highly selected group of patients in a specialized hospital (BSMMU). Follow up period was also short. Only two types of conservative measures were observed in the study. However, it is necessary to investigate a large sample and a multicenter trial of rehabilitation for a longer period of follow up.

\section{Conclusions:}

From the present study in may be concluded that to reduce pain and disability, the patients with neck pain due to cervical spondylosis may be treated effectively with rehabilitation treatment. And by this way, use of NSAIDs can be reduced and ultimately kidney disease may be decreased.

\section{Acknowledgement:}

We would like to thank the Bangladesh College of Physicians and Surgeons for sponsoring this study. We are also thankful to the patients who were gladly participated in the study.

\section{References:}

1. Bajaj $P$, Bajaj $P$, Nielsen $T G$, Nielsen $L A$. Osthoarthritis and its association with muscle hyperalgia: an experimental controlled study. Pain 2001; 93:107-114.

2. Leach JP and Davenport RJ. Neurological diseases. In: Walker BR, Colledge NR, Ralstone SH, Penman ID. Editors. Davidson's Principles and Practice of Medicine. Edinburgh. Churchill Livingstone, 2014; 26:1137-1247.

3. Barry M, Jenner J R. Pain in the neck, shoulder and arm. B M J 1995; 310:183-186.

4. Nakano KK. Neck pain In: Kelly WN. Harries ED, Rudy S, Sledge CB, editors: Text Book of Rheumatology. $3^{\text {rd }}$ ed. Philadelphia; W B Saunders 1989: 471-90.

5. Hires L F. Cervical degenerative arthritis. Post grad Med 1983; 74:123-30.

6. Loy T T. Treatment of cervical spondylosis, Med. J Aug 1983; July: 32-34.

7. Mohindra Y; Clinical and occupational aspect of cervical spondylosis, J Indian Med. Assoc. 1981; 77: 8-9 
8. Alam M N, Haq S A, Moyeenuzzaman M, Samad M A, Choudhury MKQ, Das K Ketal..Rheumatological Disorders in I P G M \& R, Bangladesh J Medicine 1996; 7:1-7.

9. Berman M B, Bausell R B. The use of nonpharmacological therapies by pain specialists. Pain 2000; 85:313-315.

10. Dieppe $P$, Szebenyl B, Evidence Based Rheumatology. The J Rheamatol. 2000: 4-6

11. Wang R Y, Yang $Y R$, Tsai M W, Wang W T, Chang $\mathrm{R}$ C. Effects of functional electric stimulation on upper limb motor function and shoulder range of motion in hemiplegic patients. Am J Phyes Med Rehabil 2002; 81 (4): 283-90.

12. Shakoor MA, Ahmed MS, Kibria G, Khan AA, Mian MAH, Hasan SA et al. Effect of Cervical Traction \& Exercise therapy on in Cervical Spondylosis. Bangladesh Medical Research Council (BMRC) Bulletin. 2002; 28 (2): 61-69.

13. Wright A, Sluka K A. Nonpharmalogical treatments for masculoskeletal pain. Clin J Pain 2001; 17 (1): 33- 46.

14. Wong A M K, Lee M Y, Chang W H Tang F T. Clinical trail of a cervical traction modality with Electromyographic Biofeedback. Am. J Phys Med Rehabil 1997; 76:19-25.

15. British Association of Physical Medicine. Pain in the neck and arm; Amulticentre trial of the effect of physiotherapy. Br Med J 1966;1: 253258.

16. Irvine D H, Foster J B, Newell D J, Klukvin B $\mathrm{N}$. Prevalence of cervical spondylosis in general practice. Lancet $1965 ; 1: 1089-92$.

17. Bhattacharjee $B N$, Islam $M Q$. Epidemiological aspects of cervical spondylosis and evaluation of conservative treatment. Bangladesh Medical Journal 2000;29 (3) 21-24.

18. Swezey R L, Swezey AM, Warner K. Efficacy of home cervical traction therapy. Am J Rehabil 1999; 78 (1) :30-2.
19. Graziano G P, Hensinger R, Patel C K. The use of traction methods to correct severe cervical deformity in rheumatoid arthritispatients: a report of five cases. Spine 2001; 26 (9): 107-81.

20. Honet C J, Purik. Cervical Radiculities : Treatment and results in 82 patients. Arch phys Med Rehabil 1976; 57 :12-16.

21. Caldwell $\mathrm{J}$ W, Krusen E M. Effectiveness of cervical traction in treatment of neck problems: Evaluation of various Methods. ArchivPhys Med Rehabail1962; 5:214-221.

22. Nichols P T R. Rehabilitation Medicine- The Management of Physical Disabilities. $2^{\text {nd }}$ ed. Butterworth \& co Ltd, London-Boston 1980: 11-31.

23. Shakoor M A, Islam M Q, Moyeenuzzaman, Mian MAH, Khan S. Effects of cervical traction and short-wave diathermy on the patients with neck pain. J Dhaka Medical College.

24. Chard J, Dieppe P. The case for Nonpharmachologic therapy of osteoarthritis. In: Cronstein B N editor. Current Rheumatology reports. Philadelphia: Current Science Inc; 2001: 88-94.

25. Tow A M. Management of myofascial pain. APLAR JRheumatol 1999; 3 (1): 372-75.

26. Tanaka S, Hachisaka K, Ogata H, Kobayashi $\mathrm{Y}$, Tanaka $\mathrm{H}$. Effect of activities of daily living on fibre type atrophy of the vastaslateralis muscle in patients with joint disorders. Am J Phys Med Rehabil 1998; 77: 122-127.

27. Singh G, Triadafilopoulos G. Epidemiology of NSAID Induced Gastrointestinal Complications. J Rheumatol 1999; 26 (56 Suppl ): 18 S-24 S.

28. Geis G S. Update on Clinical Developments with Celecoxib, a New Specific cox-2 Inhibitor: What Can We Expect? J Rheumatol 1999; 26 (56Suppl): 31S-36S. 